\title{
EXTRUSÃO ANAL DE CATETER DE DERIVAÇÃO VENTRICULOPERITONEAL-RELATO DE 2 CASOS E REVISÃO DO MANEJO
}

\section{ANALEXTRUSION OF THE VENTRICULOPERITONEALSHUNT CATHETER:TWO CASE REPORTS}

\section{Autores}

Renato C Monteiro Santos Kalínio de Kássio $O^{\circ}$ Virgílio Vilá Moura² Ronan Arnon Anchieta' Lucas Nogueira Caixeta Atahualpa Cauê Paim Strapasson

1. Médico Residente do Serviço de Neurocirurgia - Hospital Santa Rosa, CuiabáMT

2. Neurocirurgião preceptor do Serviço de Neurocirurgia - Hospital Santa Rosa, CuiabáMT

\section{RESUMO}

INTRODUÇÃO: A derivação ventriculoperitoneal (DVP) ainda consiste no tratamento para hidrocefalia mais utilizado nos tempos modernos. Kausch em 1905 foi o responsável por introduzir esse sítio como possibilidade de derivação liquórica. Apresentamos dois casos de uma complicação rara de derivação ventrículo-peritoneal que é a extrusão anal com incidência estimada entre 0,1 a 0,7\% dos casos e apresentaremos uma revisão da literatura sobre as possibilidades de abordagem dos mesmos. CASO CLÍNICO 1: Paciente feminina, 7 anos de idade, apresentou dois dias após a colocação da DVE quadro de constipação, sendo que após uso de procinéticos apresentou fezes endurecidas e extrusão do cateter distal juntamente com a válvula e o cateter ventricular. CASO CLÍNICO 2: Paciente feminina, 6 anos de idade, possuía diagnóstico pós-natal de hidroanencefalia, o pai notou a presença de exteriorização de cateter pelo ânus da paciente e procurou atendimento em serviço de urgência. Foi submetida a retirada do sistema de DVP com excisão do cateter proximal e retirada cautelosa do cateter distal extruso através do ânus sem intercorrências. A paciente apresentou deterioração clínica importante com irritabilidade e sinais de sepse. Evoluiu com choque séptico refratário e óbito após cerca de 2 meses de internação. DISCUSSÃO: Perfurações de vísceras abdominais como intestino, estômago e bexiga pelo cateter de derivação distal já foram reportados em literatura, contudo sua frequência real não é totalmente estabelecida. estima-se que a incidência da perfuração intestinal varie entre 0,1 - 0,7\% com uma mortalidade chegando a 15\%. Apesar da perfuração intestinal, menos de $25 \%$ dos casos apresentavam-se com sinais de peritonite, já 
outros $43 \%$ possuíam quadro clínico de meningite. Em nossa opinião, procedimentos invasivos para correção das perfurações intestinais pelo cateter devem ser restringidos para casos de claro abdome agudo e a conduta mais adequada deve ser a completa remoção do shunt e a derivação ventricular externa com antibioticoterapia de amplo espectro.

Palavras-chave: Derivação Ventrículo Peritoneal; Complicações Abdominais; Extrusão Anal.

\section{ABSTRACT}

INTRODUCTION: Ventriculoperitoneal (VP) shunt is still the most widely used treatment for hydrocephalus in modern era. In 1905, Kaush was responsible to start this site for liquor shunt. Here we presente two cases with a rare VP shunt complicantion wich is anal extrusion. Also we will present a literature review on the possibilities of approaching them. CLINICAL CASE 1: A 7-year-old female presented with constipation two days after External Ventricular Drain (EVD) placement. After using prokinetics she had hard stools and extrusion of the distal catheter together with the valve and the ventricular catheter. CLINICAL CASE 2: A 6-year-old female had a postnatal diagnosis of hydroanencephaly. The father noticed the presence of catheter extrusion through the patient's anus and sought care in the emergeny department. She underwent removal of the VP shunt with excision of the proximal catheter and cautious removal of the extruded distal catheter through the anus uneventfully. The patient showed significant clinical deterioration with irritability and signs of sepsis. She evolved with refractory septic shock and died after 2 months of hospitalization. DISCUSSION: Abdominal visceral perforations such as bowel, stomach and bladder by the distal catheter have already been reported in the literature, however its actual frequency has not been fully established. It is estimated that the incidence of bowel perforation lies between $0,1-0,7 \%$ with a mortality reaching $15 \%$. Despite the bowel perforation, less than $25 \%$ of the cases presented with signs of peritonitis, while another $43 \%$ had clinical meningitis. In our opinion, invasive procedures for correction of bowel perforation by catheter should be restricted to cases of clear acute abdômen and the most appropriate approach should be complete removal of the shunt and EVD placement with broadspectrum antibiotic therapy.

Key-words: Ventriculoperitoneal Shunt; Bowel Perforation; Anal extrusion.

\section{INTRODUÇÃO}

A derivação ventriculoperitoneal (DVP) ainda consiste no tratamento para hidrocefalia mais utilizado nos tempos modernos. Kausch em 1905 foi o responsável por introduzir esse sítio como possibilidade de derivação liquórica. 
As taxas de complicações variam de 17-30 \% sendo que as complicações abdominais são bastante frequentes.

Apresentamos dois casos de uma complicação rara de derivação ventrículo-peritoneal que é a extrusão anal com incidência estimada entre 0,1 a 0,7\% dos casos e apresentaremos uma revisão da literatura sobre as possibilidades de abordagem dos mesmos.

Caso clínico 1: Paciente feminina, 7 anos de idade, com diagnóstico prévio de encefalopatia hipóxico-isquêmica e hidrocefalia comunicante congênita, realizou cirurgia de derivação ventrículoperitoneal com válvula de média pressão neonatal com poucos dias de vida.

Iniciou quadro de cefaléia e rebaixamento de consciência e concomitante a esses sintomas notou abaulamento em região torácica anterior direita à qual não foi valorizada durante avaliação pediátrica inicial.

Foi recebida em serviço neurocirúrgico em escala de coma de glasgow (ECG) 3 com pupilas isocóricas e fotorreagentes e tomografia computadorizada (TC) de crânio evidenciando hidrocefalia comunicante descompensada sem a presença de cateter ventricular e a inspeção da região occipital não se verificava o "pump" da válvula.

Foi submetida a derivação ventricular externa (DVE) de urgência e o líquor coletado confirmou ventriculite com crescimento de Pseudomonas aeruginosa. Dois dias após a colocação da DVE a paciente mantinha quadro de constipação, sendo que após uso de procinéticos apresentou fezes endurecidas e extrusão do cateter distal juntamente com a válvula e o cateter ventricular. Evoluiu sem sintomas abdominais subsequentes e a TC de abdome não evidenciou sinais de pneumoperitônio.

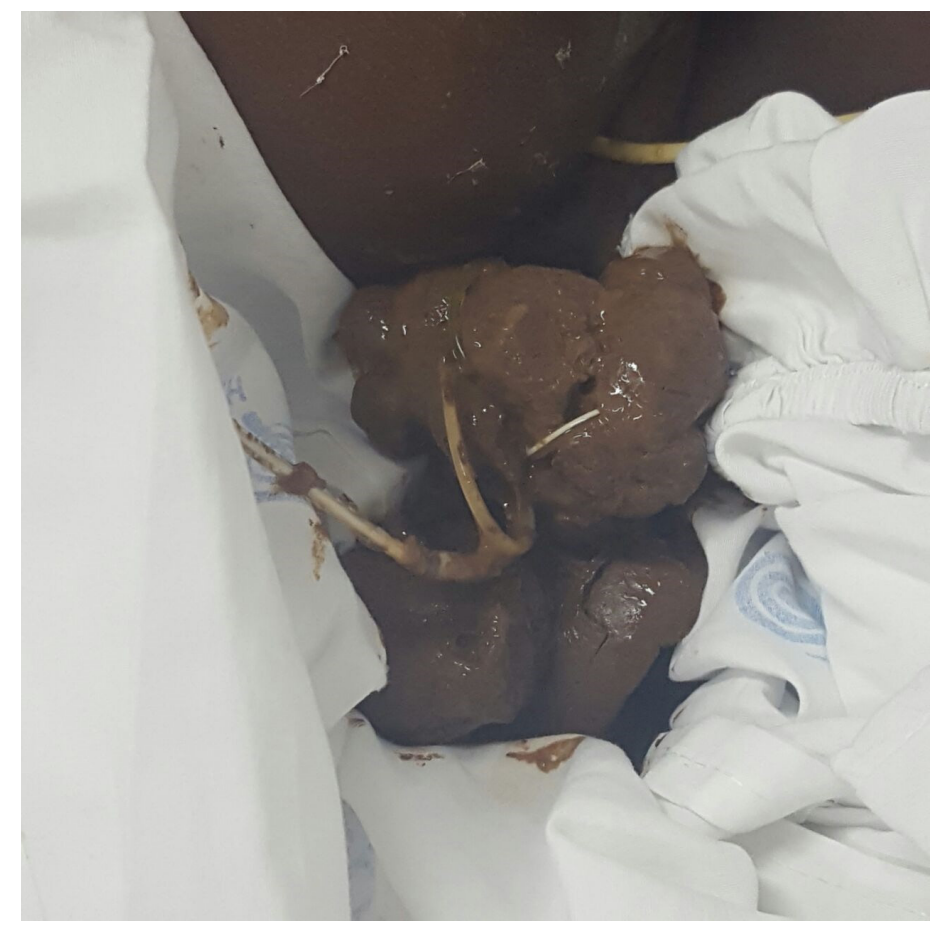

Figura 1 - Cateter proximal, Válvula e Cateter distal envolto às fezes 
Após curso de 21 dias de antibiótico e culturas negativas foi repassado DVP contralateral sem intercorrências com melhora do nível de consciência, permanecendo com disfunções cognitivas prévias e recebendo alta.

Caso clínico 2: Paciente feminina, 6 anos de idade, possuía diagnóstico pós-natal de hidroanencefalia, sendo que apresentou crescimento exagerado do crânio com grande desproporção em relação ao corpo; foi submetida a cirurgia de DVP com 2 anos de idade com estabilização do crescimento do perímetro cefálico.

O pai notou a presença de exteriorização de cateter pelo ânus da paciente e procurou atendimento em serviço de urgência. Não apresentava até o momento da admissão sintomas abdominais, ou mesmo alterações clínicas como febre e piora do comportamento.

Os exames de imagem confirmaram a manutenção do cateter ventricular e válvula bem posicionados. Foi submetida a retirada do sistema de DVP com excisão do cateter proximal e retirada cautelosa do cateter distal extruso através do ânus sem intercorrências. Foi mantida com DVE sendo que o líquor se apresentava com características purulentas e a cultura posteriormente revelou crescimento de Staphylococus epidermidis.

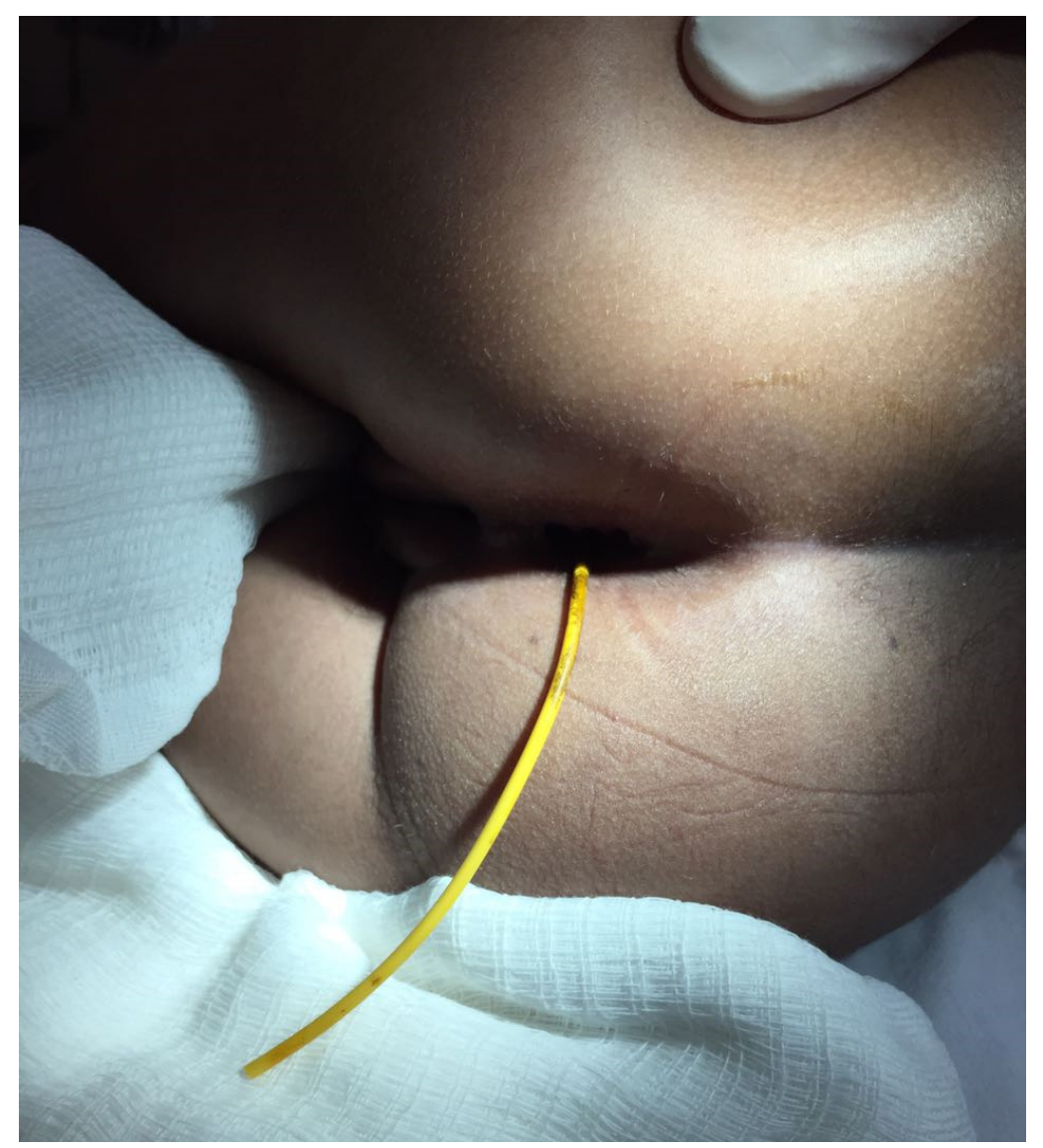

Figura 2 - Cateter distal exteriorizando-se pelo ânus 
Recebeu antibioticoterapia guiada com troca do sistema DVE diversas vezes devido ao mau funcionamento secundário ao conteúdo espesso do líquor. A paciente apresentou deterioração clínica importante com irritabilidade e sinais de sepse. Evoluiu com choque séptico refratário e óbito após cerca de 2 meses de internação.

\section{DISCUSSÃO}

Perfurações de vísceras abdominais como intestino, estômago e bexiga pelo cateter de derivação distal já foram reportados em literatura, contudo sua frequência real não é totalmente estabelecida. estima-se que a incidência da perfuração intestinal varie entre 0,1 - 0,7\% com uma mortalidade chegando a $15 \%$.

Apesar da perfuração intestinal, menos de $25 \%$ dos casos apresentavam-se com sinais de peritonite, já outros $43 \%$ possuíam quadro clínico de meningite.

Ghritlaharey em sua série de 10 casos não encontrou nenhum dos seus paciente com sinais de peritonite durante ou após a remoção e revisão do shunt.

Tais condições inferem que a possível patogênese da perfuração ocorreriam de forma crônica. Pacientes jovens são mais acometidas possivelmente devido a fragilidade da parede intestinal, a qual pode ser mais facilmente penetrada pela porção distal do cateter de DVP. Os movimentos peristálticos seriam os responsáveis pela migração caudal do sistema.

Akyuz et al propôs que a ponta do cateter adere a parede da víscera e a pressão e contato constante juntamente com reações inflamatórias locais levam a erosão da parede da víscera e entrada da ponta do cateter no lúmen.

A rápida identificação da disfunção minimiza os riscos infecciosos. o cateter distal deve ser removido de maneira menos invasiva possível e sempre deve ser confirmado a presença de meningite para o correto tratamento infeccioso e posterior reintrodução do shunt.

Nossos dois casos apresentados não evidenciaram sinais de peritonite, assim como a maioria das séries relatadas pela literatura.

Filho et al, expôs um caso em que o paciente foi submetido a laparatomia exploradora com correção da perfuração intestinal e a porção do cateter que não havia penetrado o intestino foi mantida no espaço peritoneal após confirmação da ausência de infecção. Contudo houve crescimento de bactérias gram-negativas no líquor 5 dias após o procedimento e o shunt necessitou ser retirado.

Em nossa opinião, procedimentos invasivos para correção das perfurações intestinais pelo cateter devem ser restringidos para casos de claro abdome agudo e a conduta mais adequada deve 
ser a completa remoção do shunt e a derivação ventricular externa com antibioticoterapia de amplo espectro.

Não há dados que contra-indiquem a repassagem do cateter para o peritônio quando possível. Grande parte dos casos expostos aguardavam a presença de pelo menos 2-3 culturas de líquor negativas, e o tempo médio de antibioticoterapia foi de 10 dias.

\section{REFERÊNCIAS}

1 - Snow RB, Lavyne MH, Fraser R. Colonic perforation by ventriculoperitoneal shunts. Surg Neurol 1986;25:173-7.

2 - Akyuz M, Ucar T, Goksu E. A thoracic complication of ventriculoperitoneal shunt: Symptomatic hydrothorax from intrathoracic migration of a ventriculo-peritoneal shunt catheter. $\mathrm{Br} \mathrm{J}$ Neurosurg. 2004;18:171-3.

3 - Ghritlaharey KR, Budhwani KS, Shrivastava DK, Gupta G, Kushwaha AS, Chanchlani R, Nanda, M. Trans-anal protrusion of ventriculo-peritoneal shunt catheter with silent bowel perforation: report of ten cases in children. Pediatr Surg Int. 2007; 23:575-580.

4 - Teegala R, Kota LP. Unusual complications of ventriculo peritoneal shunt surgery. J Neurosci Rural Pract. 2012;3(3):361-364. doi:10.4103/0976-3147.102628

5 - Filho PMM, D’Agostini D, Silva RS, Junior NDA, Winkelmann L, Diego Zambonin D.- Bowel Perforation and extrusion of a ventriculo-peritoneal shunt through the anus. Brazilian Journal of Neurosurgery 24 (1): 69-74, 2013

6 - Sarkari A, Borkar SA, Mahapatra AK. Anal extrusion of migrated ventriculo-peritoneal shunt catheter: An unusual complication and review of literature. Asian J Neurosurg. 2016;11(4):459. doi:10.4103/1793-5482.150002

7 - Hasan A, Sharma S, Chopra S, Purohit DK. Anal extrusion of ventriculoperitoneal shunt: A report of two cases and review of literature. J Pediatr Neurosci 2018;13:8-12

8 - Thiong'o, G., Luzzio, C., \& Albright, A. (2015). Ventriculoperitoneal shunt perforations of the gastrointestinal tract, Journal of Neurosurgery: Pediatrics PED, 16(1), 36-41. Retrieved Oct 27, 2019, from ttps://thejns.org/pediatrics/view/journals/j-neurosurg-pediatr/16/1/article-p36.xml 